\title{
Metabolic and coagulation effects of citrate: down to the last detail!
}

\author{
Patrick M. Honore*, Rita Jacobs, Inne Hendrickx, Elisabeth De Waele, Viola Van Gorp and Herbert D. Spapen \\ See related research by Slowinski et al., http://www.ccforum.com/content/19/1/349
}

The recently published Liver Citrate Anticoagulation Threshold (L-CAT) trial convincingly showed that continuous renal replacement therapy with regional citrate anticoagulation (CRRT-RCA) was safe and effective in patients with severely impaired liver function. Striking findings were a relatively low incidence of acid-base disorders and a markedly long 72-h filter survival [1]. Nonetheless, we would like to comment on two important metabolic issues. First, the low (2\%) incidence of severe metabolic alkalosis, defined as a $\mathrm{pH}>7.55$, is probably highly underestimated. Indeed, we recently evaluated the occurrence of metabolic alkalosis defined either as a $\mathrm{pH}>7.50$ or as an apparent strong ion difference (SIDa) $>45 \mathrm{mmol} / \mathrm{L}$ according to the Stewart-Figge methodology [2] in patients undergoing CRRT-RCA with an isonatremic low-chloride containing diluted citrate solution. Calculating SIDa revealed a ninefold increase in the percentage of metabolic alkalosis [3]! Accordingly, applying the SIDa approach likely will uncover a substantially higher incidence of severe metabolic alkalosis in the L-CAT patients. Second, the LCAT investigators attributed the long filter lifespan to a more automated fine-tuning of citrate and calcium dosing. However, we recently demonstrated that keeping post-filter ionized calcium (iCa) within tight limits (i.e., 0.2-0.3 $\mathrm{mmol} / \mathrm{L}$ ) during CRRT-RCA resulted in a 72-h filter survival comparable with that observed in the LCAT trial [4]. Post-filter iCa levels in all L-CAT trial groups were within the same range and thus may have accounted for a better preserved filter patency.

\section{Authors' response}

Torsten Slowinski and Detlef Kindgen-Milles

We agree with Honoré et al. that the use of the StewartFigge approach probably would have discovered a higher incidence of alkalosis in the study population of the L-CAT study [1]. However, in clinical practice as well as in other studies investigating RCA the Henderson and Hasselbalch approach is used. For practical reasons in this multicenter trial and to allow better comparisons with other studies, we decided to use the latter approach in the L-CAT study.

The major outcome parameter for safety in the L-CAT study was the incidence of severe acidosis $(\mathrm{pH} \leq 7.2)$ or alkalosis $(\mathrm{pH} \geq 7.5)$ in patients with different degrees of liver failure. Most important, we found no significant difference between these groups and this was true not only for $\mathrm{pH}$, but also for bicarbonate concentration. Thus, even if the overall incidence of

\footnotetext{
* Correspondence: Patrick.Honore@az.vub.ac.be

ICU Department, Universitair Ziekenhuis Brussel, Vrije Universiteit Brussel, 1090 Brussels, Belgium
}

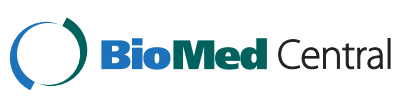

(c) 2015 Honore et al. Open Access This article is distributed under the terms of the Creative Commons Attribution 4.0 International License (http://creativecommons.org/licenses/by/4.0/), which permits unrestricted use, distribution, and reproduction in any medium, provided you give appropriate credit to the original author(s) and the source, provide a link to the Creative Commons license, and indicate if changes were made. The Creative Commons Public Domain Dedication waiver (http://creativecommons.org/publicdomain/zero/1.0/) applies to the data made available in this article, unless otherwise stated.

alkalosis might have been slightly different using the Stewart-Figge approach, most probably no differences between the study groups would have been detected this way.

Regarding the problem of metabolic alkalosis during RCA, it is of note that other colleagues, i.e., Oudemannsvan Straaten et al. [5], reported a significantly higher incidence of metabolic alkalosis (bicarbonate $>30 \mathrm{mmol} / \mathrm{L}$ ) in patients treated with conventional bicarbonate containing solutions in systemic anticoagulated continuous venovenous hemofiltration $(\mathrm{CVVH} ; 26 \%)$ compared with those who were treated with RCA-CVVH (9\%). This observation clearly shows an urgent need to analyze different protocols in CRRT with regard to their efficiency to control acid-base state.

Of note, we agree that keeping ionized calcium concentrations within the extracorporeal circuit in tight limits is the most important task in terms of avoiding filter clotting in RCA-CRRT. In vitro data showed that $\mathrm{iCa}$ 
has to be lowered to at least $<0.4 \mathrm{mmol} / \mathrm{L}$ to provide sufficient anticoagulation [6].

Discussing filter lifetime as efficacy endpoint, the LCAT study showed a filter patency rate of $>90 \%$ after 72 hours if treatment stops not caused by clotting were censored. Thus, the filter clotting rate has decreased continuously compared with earlier publications of Morgera et al. [7] and Kalb et al. [8], which used exactly the same RCA protocol. Obviously, with increasing experience and training of staff, clotting events can nowadays be avoided almost completely. This way, safe and effective CRRT can be applied and delivery failure is avoidable.

\section{Abbreviations}

CRRT: continuous renal replacement therapy; CRRT-RCA: continuous renal replacement therapy with regional citrate anticoagulation; $\mathrm{CWH}$ : continuous veno-venous hemofiltration; iCa: ionized calcium; L-CAT: Liver Citrate Anticoagulation Threshold; RCA: regional citrate anticoagulation; SIDa: apparent strong ion difference.

\section{Competing interests}

The authors declare that they have no competing interests.

\section{Authors' contributions}

PMH and HDS designed the study. PMH, RJ, IH, EDW, WG and HDS participated in drafting the manuscript. All authors have read and approved the final manuscript.

Published online: 12 December 2015

\section{References}

1. Slowinski T, Morgera S, Joannidis M, Henneberg T, Stocker R, Helset E, et al. Safety and efficacy of regional citrate anticoagulation in continuous venovenous hemodialysis in the presence of liver failure: the Liver Citrate Anticoagulation Threshold (L-CAT) observational study. Crit Care. 2015;19:349.

2. Egi M, Naka T, Bellomo R. The acid-base effect of changing citrate solution for regional anticoagulation during continuous veno-venous hemofiltration. Int J Artif Organs. 2008;31:228-36.

3. Jacobs R, Honore PM, Spapen HD. Some metabolic issues should not be neglected when using citrate for continuous renal replacement therapy! Crit Care. 2015;19:50.

4. Jacobs R, Honoré PM, Bagshaw SM, Diltoer M, Spapen HD. Citrate formulation determines filter lifespan during continuous veno-venous hemofiltration: a prospective cohort study. Blood Purif. 2015;40:194-202.

5. Oudemans-van Straaten HM, Bosman RJ, Koopmans M, van der Voort PH, Wester JP, van der Spoel J, et al. Citrate anticoagulation for continuous venovenous hemofiltration. Crit Care Med. 2009;37(2):545-52.

6. Calatzis A, Toepfer M, Schramm W, Spannagl M, Schiffl H. Citrate anticoagulation for extracorporeal circuits: effects on whole blood coagulation activation and clot formation. Nephron. 2001;89(2):233-6.

7. Morgera S, Schneider M, Slowinski T, Vargas-Hein O, Zuckermann-Becker H, Peters $\mathrm{H}$, et al. A safe citrate anticoagulation protocol with variable treatment efficacy and excellent control of the acid-base status. Crit Care Med. 2009;37(6):2018-24.

8. Kalb R, Kram R, Morgera S, Slowinski T, Kindgen-Milles D. Regional citrate anticoagulation for high volume continuous venovenous hemodialysis in surgical patients with high bleeding risk. Ther Apher Dial. 2013;17(2):202-12. 